\title{
Constraint-induced movement therapy for moter recovery after stroke
}

\author{
David M. Morris ${ }^{\mathrm{a}, *}$, Jean E. Crago ${ }^{\mathrm{b}}$, Stephanie C. DeLuca ${ }^{\mathrm{c}}$, Rama D. Pidikiti ${ }^{\mathrm{d}}$, \\ Edward Taub ${ }^{\mathrm{e}}$ \\ ${ }^{a}$ Division of Physical Therapy, 900 19th St. South, RM 102, University of Alabama at Birmingham, Birmingham, \\ AL 35294, USA \\ ${ }^{\mathrm{b}}$ Spain Rehabilitation Center, University of Alabama at Birmingham, Birmingham, AL, USA \\ ${ }^{\mathrm{C}}$ University of Alabama at Birmingham, Birmingham, AL, USA \\ ${ }^{\mathrm{d} D i r e c t o r}$ of Rehabilitation, Veterans Administration Medical Center, Birmingham, AL, USA \\ 'Department of Psychology, University of Alabama at Birmingham, Birmingham, AL, USA
}

\begin{abstract}
A new therapeutic approach to rehabilitation of movement after stroke, termed Constraint-Induced (CI) Movement Therapy, has been derived from basic research with monkeys given somatosensory deafferentation. CI consists of a family of therapies; their common element is that they induce stroke patients to greatly increase the use of a more affected upper extremity for many hours a day over a 10-14 consecutive-day period. These therapies have significantly improved quality of movement and substantially increased amount of use of a more affected extremity in the activities of daily living in the life situation. The purpose of this paper is to describe the protocol used by the investigative team that developed the family of $\mathrm{CI}$ therapies and examined them as an effective rehabilitation approach. () 1997 Elsevier Science Ireland Ltd.
\end{abstract}

Keywords: Stroke; Rehabilitation; Therapy; Upper extremity; Motor control; Behavior

\section{Introduction}

Since 1986, a technique termed ConstraintInduced (CI) Movement Therapy, has been de-

\footnotetext{
* Corresponding author. Tel.: +1205 9343566; fax: +1205 9757787.
}

veloped and researched. This therapeutic approach substantially enhances the quality of movement and (especially) the amount of use of the more affected upper extremity (UE) of chronic stroke patients when performing activities of daily living in the home situation [1-5]. Since no current technique appears to enable full transfer of rehabilitation gains into the life situation, it was 
believed that rehabilitation professionals would benefit from a full description of the protocol used when treating patients with a CI approach. This may be particularly true in the event that other clinicians and investigators are interested in duplicating the results or employing this therapy effectively. Therefore, the main purpose of this article is to describe the CI protocol used in this laboratory at The University of Alabama at Birmingham (UAB).

In their 1979 article 'Stroke Recovery; He can but Does He?', Andrews and Stewart [6] noted a difference between patients' motor capabilities in the rehabilitation clinic and what they actually do in their living situation. Activities of daily living they observed in the clinic were not performed as well in the home situation in $25-45 \%$ of cases. Many rehabilitation professionals anecdotally support these findings as they often fail to see carry over of therapeutic gains made in the clinic to their patients' everyday life situation. Virtually no attention has been directed toward this commonly observed phenomenon in the rehabilitation literature, yet its ramifications toward motor recovery are significant. To further extend this pessimistic view of stroke rehabilitation, physical rehabilitation professionals commonly believe that patients reach a plateau in motor recovery, usually within 6-12 months after stroke [7,8]. Advocates of this notion, believe that rehabilitation efforts administered after this time have little chance for success. Several investigators have presented data to support the view that improvements in motor function are possible after this time $[9,10]$. Likewise, studies conducted regarding CI, suggest that motor recovery can occur later than 12 months after a stroke [1-5]. In order to provide a context for understanding CI therapy and the protocol we employ, it is helpful to describe the way the therapy developed.

\section{Research with monkeys}

Research exploring the effects of somatosensory deafferentation in monkeys has demonstrated that if a single monkey forelimb is deafferented, the monkey will not use that extremity in the free situation [11,12]. Use of the deaffer- ented extremities can be regained however, if the intact limb is restrained and the monkey is 'forced' to use the extremity. Additionally, it was demonstrated that this return in function could be permanent if the restraint was maintained for a period of 1-2 weeks. Movement restriction for shorter periods of time, 1 or 2 days, failed to produce permanent change in motor behavior.

Further studies demonstrated that certain training procedures can also be used to enable monkeys to regain use of their deafferented limbs [11-18]. One type of training, conditioned response technique, demonstrated little effectiveness in producing transfer of limb use to the life situation. However, when another training procedure, termed shaping, was employed, substantial improvements of motor function in the life situation was observed. Shaping procedures are techniques that approach a desired motor outcome in small, successive steps [19-23]. This approach allows subjects to experience successful gains in performance with relatively small amounts of motor improvement. Shaping is described in more detail later in this paper.

Observations drawn from these studies led to the development of a hypothesis to explain why the restraint and training procedures improve motor recovery after deafferentation. The theory, named learned non-use, was first described by Taub, and is believed to extend to humans following central nervous system damage [13-16].

Following substantial neurological injury, a shock-like phenomenon results in a dramatically depressed condition of motoneurons. Experimentally-induced deafferentation creates a similar condition in monkeys. Whether at the level of the spinal cord (spinal shock) or brain (diaschisis of cortical shock), the resulting neural shock renders the subject unable to perform motorically. Over time, however, recovery processes that are not well understood, improve the ability of the central nervous system to, at least potentially, produce movement. During the period of neural shock, the monkey, being unable to move the deafferented limb, learns to compensate for function with the uninvolved limb. As neural shock resolves and neural function starts to improve, attempts to use the deafferented limb result in clumsy inef- 


\section{DEVELOPMENT OF LEARNED NONUSE}

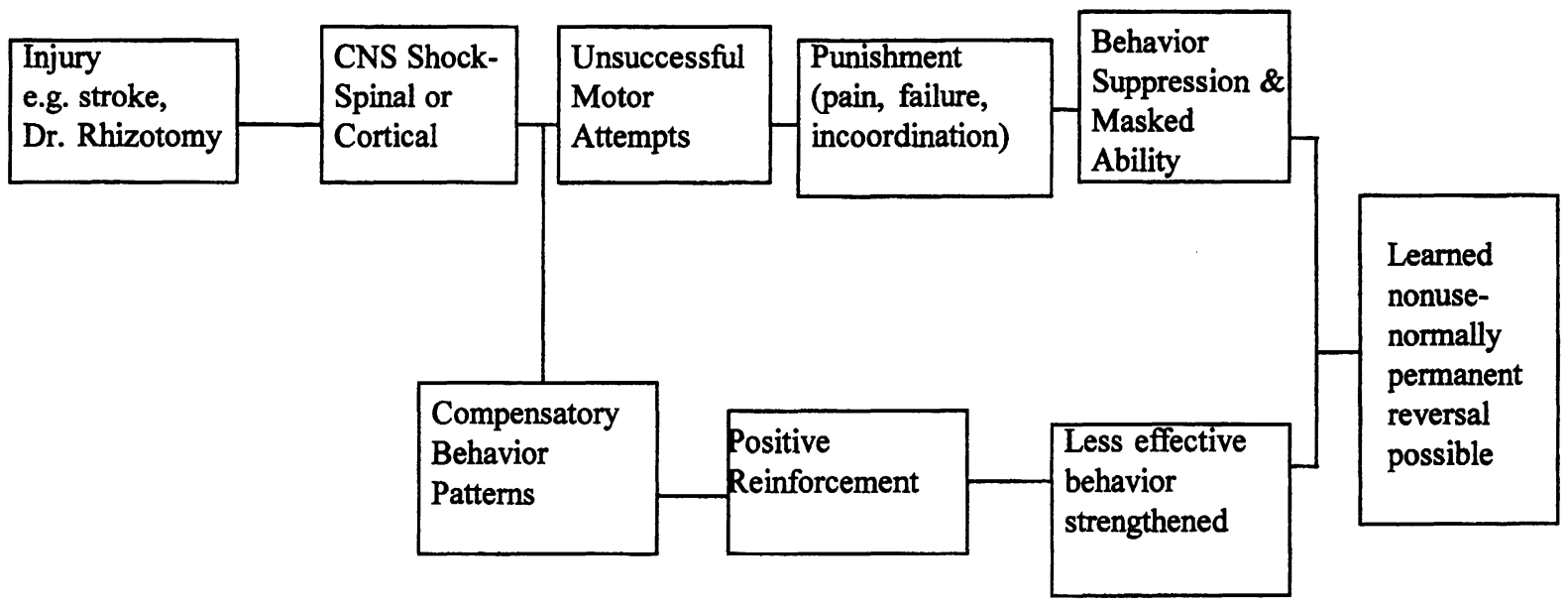

Fig. 1. Schematic model for development of learned non-use.

ficient movement (i.e. dropping food, falling). The resulting motor outcome punishes use of the deafferented limb and positively reinforces compensation with the uninvolved limb. In effect, the monkey 'learns not to use' the more affected limb (Fig. 1).

When techniques are applied, learned non-use can be overcome. Restriction of the intact limb necessitates the use of the affected limb for nor- mal activities. When used for several days or longer, the restraint leads to a permanent reversal of the learned non-use phenomenon (Fig. 2). Likewise, shaping leads to overcoming learned non-use as it positively reinforces the previously punished attempted use of the affected limb.

Direct tests of the learned non-use theory were conducted with monkeys. One study involved restraining the deafferented limbs of monkeys for 3

\section{OVERCOMING LEARNED NONUSE}

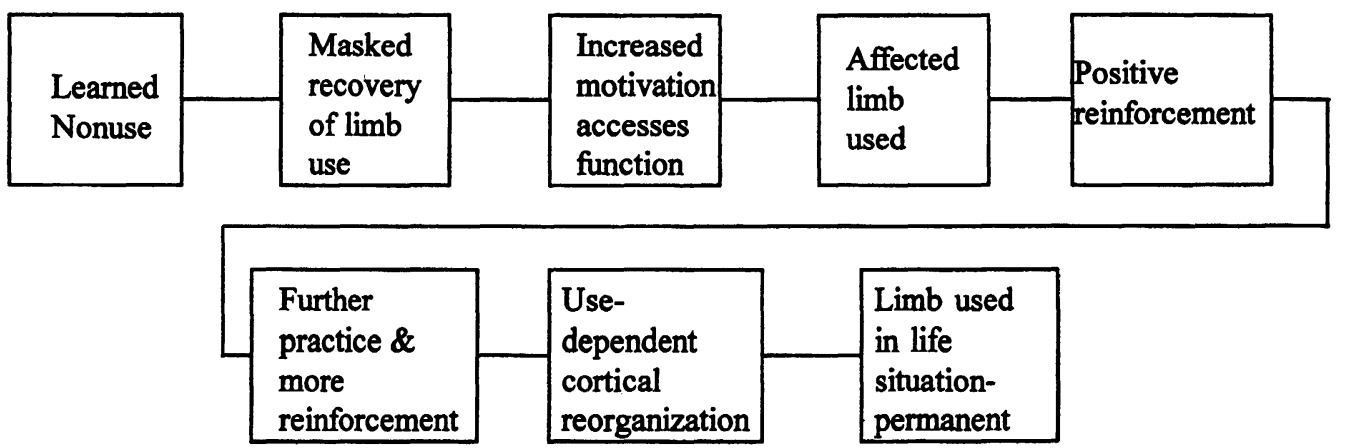

Fig. 2. Schematic model of mechanism for overcoming learned non-use. 
months following surgery $[13,14]$. These procedures prevented use of the deafferented limb during the neural shock period and thus, according to the formulation, should have prevented learned non-use from developing. In conformity with this prediction, when the restraining device was removed, the monkeys spontaneously used the deafferented limb in a functional manner. Results from this study confirm the learned nonuse hypothesis.

Another direct test of the learned non-use phenomenon involved prenatal deafferentation of monkeys [25]. Three animals received forelimb deafferentation during the prenatal period; two at two-thirds of the way through gestation and one at two-fifths of the way through gestation. Results from one of the animals was unclear due to early illness and muscular deformity. For the other two animals, purposive use of the deafferented limb was exhibited from the first day of extrauterine life (i.e. used for postural support during 'sprawling' and in pushing to a sitting position). Later these early-deafferented animals developed almost all species-characteristic movement patterns, though they were performed clumsily as a consequence of the absence of somatosensation.

The learned non-use hypothesis could provide at least a partial explanation for typical motor recovery in humans following a stroke. Instead of spinal shock, such as that experienced by the deafferented monkeys, a stroke patient would experience cortical shock or diaschisis. The learned non-use mechanism would be applicable in all other aspects and could account for a lack of transfer from clinical gains into the home setting. Likewise, a logical approach to overcoming learned non-use with humans following stroke would be to apply $\mathrm{CI}$ therapy in a manner similar to that found to be effective for the deafferented monkeys.

\section{Studies with humans}

Wolf et al. [5] carried out the first attempts to apply CI techniques to improve UE motor recovery after stroke. Twenty-five subjects were recruited for participation; all had experienced brain damage at least 1 year prior to participation.
Sixteen had experienced a stroke and five had experienced traumatic brain injury. Based on previous studies using EMG biofeedback, the researchers chose only subjects possessing voluntary finger and wrist extension while still demonstrating significant disability. They believed that these subjects would be more amenable to the rehabilitation procedures. Subjects wore a sling on their affected UE for a period of 2 weeks. The sling was worn during all waking hours except for a period of $0.5 \mathrm{~h}$ of exercise daily, forcing use of the more affected UE. The sling was removed during sleep. No specific training technique was combined with the restraint procedure. A battery of 21 motor tasks were used to assess the effectiveness of treatment. This evaluation method has since been modified and is presently used as the Wolf Motor Function Test (WMFT) - described later in this paper. The test was administered 6-10 hours before intervention, 1 week into the 2-week intervention period, immediately after intervention, and four times during the following year. Results demonstrated significant improvements in 19 of 21 tasks on the WMFT regarding force or time-based measures. These improvements were maintained, and sometimes enhanced, throughout the follow-up period. However, quality of movement ratings did not demonstrate significant changes Regardless, the investigators believed that their findings supported the existence of the learned non-use phenomenon and the notion that forced use can reverse its effects.

Taub [26] began applying CI therapy to human subjects recovering from stroke with a pilot experiment conducted with nine chronic stroke patients. These subjects had experienced CVAs from 1 to 18 years prior to participation and were required to meet inclusionary criteria similar to those used by Wolf et al. [5] (i.e. possessing some wrist and finger extension while still demonstrating significant disability). Additionally, subjects had to demonstrate good balance as they would be wearing a sling and unable to use their stronger upper extremity to protect themselves in the event of a fall. Subjects were randomly assigned to either an experimental group (four subjects) or an attention-control group (five subjects). Those assigned to the experimental group wore a sling on the less 
involved upper extremity for a period of 12 days. During this time, subjects wore the sling during all waking hours except when specific activities were being carried out (i.e. those where it may be unsafe or unreasonably difficult to use the more affected upper extremity exclusively). Additionally, a behavioral contract was used to elicit agreement from the subject to wear the restraint device for at least $90 \%$ of their waking hours during the intervention period. This behavioral contract specifically identified activities when the subject is to use the more involved UE exclusively, to use both UEs, and, for safety reasons, to use the less affected UE. The contract is still used in the present CI protocol and is described in more detail later in this paper.

Attention-control subjects were guided to focus attention on their more affected UE in three ways: (1) they were encouraged to try using their more involved UE with as many new activities as possible at home. Activity was recorded and monitored throughout the 2-week period; (2) subjects received two sessions (labeled physical therapy) involving activities requiring neither active movement or limbering of the more involved UE; and (3) patients were given self range-of-motion exercises to be carried out at in which the affected extremity was moved passively be the less involved UE.

Treatment effectiveness was assessed by three methods; two laboratory tests of motor function (the WMFT and Arm Motor Activity Test AMAT), and a structured interview exploring functional use in the life setting - the motor activity log (MAL). The AMAT differs from the WMFT in that it consists of more complex tasks involving complete activities of daily living. Both tests assessed performance time, functional ability, and quality of movement for all tasks examined. Two six-point scales were developed to rate functional ability and quality of movement. The AMAT has since been dropped from this laboratory's battery of tests because it yielded similar information as the WMFT, and was believed to be less sensitive than the WMFT in the CI intervention, and was more difficult to administer than the WMFT. However, the AMAT is still used in other laboratories investigating
Table 1

Definition of constraint induced interventions for stroke patients by group designation

\begin{tabular}{lll}
\hline Group & Intervention & $N$ \\
\hline 1 & $\begin{array}{l}\text { Sling constraint of the contralateral upper } \\
\text { extremity and supervised task practice with } \\
\text { the affected arm (but no explicit training) }\end{array}$ \\
2 & $\begin{array}{l}\text { Attention control procedures } \\
3\end{array}$ & $\begin{array}{l}\text { Sling constraint of the contralateral arm } \\
\text { plus shaping of movements of the affected } \\
\text { arm }\end{array}$ \\
4 & $\begin{array}{l}\text { Half-glove on the contralateral hand as a } \\
\text { reminder not to use it plus shaping of } \\
\text { movements of the affected arm }\end{array}$ \\
& $\begin{array}{l}\text { Shaping of movements of the affected arm } \\
\text { only (no constraint of contralateral arm } \\
\text { movement) }\end{array}$ \\
5 & $\begin{array}{l}\text { Intensive physical therapy administered } \\
\text { (6 h/day for 10 consecutive weekdays) }\end{array}$ \\
\hline 6
\end{tabular}

CIT [27]. This project marked the first time that the MAL was used. The MAL continues to be used by this laboratory and is described in greater detail later in this paper.

Results demonstrated a substantial and significant improvement in motor function (both the WMFT and AMAT) with the experimental subjects. In contrast, no significant gains were seen in motor function with the attention-control subjects. Similarly, MAL scores did not significantly improve with the attention-control subjects. Experimental subjects, however, experienced an improvement of almost 2.5 rating steps (out of 6) $(P<0.0001)$ in MAL scores. More importantly, these treatment gains were fully maintained by experimental subjects 2 years after intervention.

\section{More recent research with human subjects}

The pilot experiment just described has since been extended to include 19 additional subjects using four different modified treatment protocols (see Table 1) [2-4]. With the exception of modifying the treatment protocol, recent studies have been conducted in a similar manner and with 
essentially the same investigative team as with earlier work. One exception is that the AMAT was dropped from the project's battery of tests. A more detailed description of the modified treatment protocols and their effectiveness follows.

\subsection{Group 3 - sling constraint plus shaping}

The training procedure of shaping was added to the treatment protocol for five experimental subjects [28]. A more detailed description of shaping is provided later in this paper. Shaping tasks selected were created and tailored for each subject's intervention program. Results of this study indicated that this intervention produces no greater an effect than the sling plus supervised practice protocol (Group 1) employed in the original experiment [1]. This finding was surprising to the investigative team as they expected to find an additive effect of combining the two constraint techniques (sling and shaping) identified as effective when used separately in previous monkey studies. After considering the results of this study, two possible explanations have been offered for the lack of difference between groups 1 and 3 . First, subjects in group 1 experienced such a high degree of improvement (e.g. mean post-treatment scores on the MAL of 4 - 'almost normal') that it may be unreasonable to expect a greater improvement with even a more superior form of intervention. Second, in some ways, supervised task practice, as is used by most therapy professionals, is a form of shaping. Therefore the two techniques may not render significantly different results when used in combination with sling intervention.

\subsection{Group 4 - half glove plus shaping}

Subjects in this group wore a half glove on the less affected UE for $90 \%$ of their waking hours instead of a sling. The half glove, unlike the sling, did not restrict use of the less affected UE. Instead, it served merely as a reminder to the subject to use the more affected UE with functional tasks. The main advantage to this modification is that the half glove allows use of the less affected UE in necessary situations (i.e. when the patient is propelling a wheelchair or needs to steady themselves to prevent a fall). This procedure allows easy extension of CI therapy to a larger group of subjects where these considerations are of concern. Study results indicated that the use of the half glove rendered equally improved motor function and amount of use as groups 1 and 3 in the short term, but not 2 years after the end of treatment.

\subsection{Group 5 - shaping only}

Subjects in group 5 were subjected to shaping procedures only for $6 \mathrm{~h} /$ day for 10 consecutive weekdays and did not wear any form of restraint or reminder on the less affected UE. They were, however, encouraged to use the more affected UE outside of the clinic and they kept a diary regarding these attempts. Results indicated that shaping only did significantly improve use of the limb in both the clinic and life situation. Motor function tests indicated that they improved comparably to subjects in the constraint groups 1,3 , and 4. However, MAL results indicated that the shaping only protocol did not carry over into the life situation as well as the constraint protocols.

\subsection{Group 6 - intensive physical therapy}

Several studies have indicated that conventional physical therapy does not produce meaningful improvement in chronic stroke patients (summarized in $[7,8,26]$. Subjects in group 6 received intensive physical therapy over 10 consecutive weekdays. They received six contact hours with project staff daily consisting of $1 \mathrm{~h}$ of aquatic physical therapy, two 45-min sessions of conventional physical therapy (proprioceptive neuromuscular facilitation, progressive resistance and isokinetic exercise, cardinal plane movements, etc.), two 45-min sessions of task practice, a lunch break, and appropriate rest intervals. Both aquatic and conventional physical therapy sessions were tailored to the subjects' individual needs and aimed at improving motor control and functional use of the more affected upper extremity. Results demonstrated improvements on all measures comparable to the sling plus shaping group (Group 
3). These results refute earlier studies regarding the effectiveness of physical therapy for chronic stroke; at least when delivered in an intensive fashion with subjects meeting our inclusionary criteria. Additionally, it provides information about comparability of CI to more traditional modes of physical rehabilitation. We believe that CI may be preferable to the intensive physical therapy protocol as it is less labor intensive and more likely to be cost effective while rendering similar results.

\section{The CI protocol}

\subsection{Inclusionary criteria}

At present, the UAB laboratory is recruiting subjects for three CI research projects. Each of the three have different inclusionary criteria that consider subjects' movement and balance capabilities. The same screening evaluation form is used for all three projects. The specific inclusionary criteria are described below.

\subsubsection{Spain rehabilitation center $(S R C)$ project}

Subjects enrolled in this project must be able to extend $20^{\circ}$ at the wrist and $10^{\circ}$ at the fingers, yet demonstrate significant disability. These criteria are identical to those used in previous CI studies conducted by our investigative team. Patients must also demonstrate good balance as evidenced by the ability to withstand moderate perturbations in standing, walk safely with a sling on, independently rise from a chair (with or without using the more affected UE), and walk up/down two stairs consecutively. Subjects must demonstrate good balance for safety reasons as they will be wearing a sling for extended periods of time throughout the day and while unsupervised at home.

\subsubsection{National institutes of health (NIH) project}

The UAB research team is also assisting a research team led by Dr. Leonard Cohen in the Human Motor control Branch of NIH and Dr. M. Lynn Grber of the Department of medical Rehabilitation of the NIH Clinical Center in Bethesda, MD to identify research subjects for a similar CIT research project. Subjects for this study must meet the same minimal motor criteria yet do not need to meet the stringent balance criteria established for the SRC subjects. Subjects in the NIH projects are admitted as inpatients during the treatment period and are always supervised. Therefore, even patients confined to a wheelchair can participate in this project.

\subsubsection{Veteran's administration (VA) medical center project}

A new project is in the pilot phase at the VA Medical Center in Birmingham, AL. This project is being conducted by the same investigative team as the first project. This work involves recruiting subjects who do not meet the minimal motor criteria established for the SRC and NIH CI projects. Instead, new motor criteria have been established using a modified version of the UE portion of the Fugl-Meyer Test of Motor Function. This project is particularly important as it extends the research to much more disabled individuals than have been previously included. The project staff expects that many of the subjects appropriate for this project will also have significant problems with balance. Therefore, new balance criteria have been established for this group of subjects. Since subjects with balance deficits will be accepted into this project, activities outside of the clinic may need to be modified.

\subsection{Subject selection}

The recruiting efforts by project staff have produced an overabundance of potential subjects for the project. These efforts have included presentations at a local stroke club meeting, advertisements in local Birmingham newspapers, letters sent to local physicians, flyers distributed at Senior Citizen Community Centers and local churches. More recently, nationally distributed news stories and television segments have led to numerous phone calls from willing participants nationwide.

When a client first contacts the project staff, a telephone screening protocol is administered. Project staff are guided to conduct this interview according to a written protocol narrative. The project staff member questions the client regard- 
ing their balance (e.g. history of falls), presence of serious medical conditions (e.g. uncontrolled high blood pressure, diabetes), and medications prescribed. The potential subject is then asked to sit in a chair with arm rests and place their more affected UE such that their hand hangs off the front of the chair arm rest. They are then asked to perform extension at the wrist and fingers to gain information about their ability to meet the different project's motor criteria. If they apparently meet the inclusionary criteria, they are asked about their ability to use their more affected UE to accomplish everyday functional tasks. These task items are taken from the MAL (discussed in more detail below). The telephone survey protocol includes statements reminding the interviewer that appropriate study subjects will be those providing responses in the mid-range of options. For example, 'someone who can use their arm for practically nothing is probably not appropriate for this study; just as someone who can accomplish most of the tasks with a fair amount of ease is probably not appropriate'. Subjects who appear to meet one of the three project's inclusionary criteria are scheduled for a more thorough screening evaluation at the research laboratory.

The clinic screening evaluation is conducted by a physician, physical therapist, and research assistant. The physicians' portion of the screening evaluation consist of a general systems review to identify serious medical problems (e.g. uncontrolled hypertension). If such problems are identified, subjects are advised to contact their usual physician and told that they may be considered for enrollment into one of the projects if the medical problem is resolved. The physical therapists' portion of the screening evaluation is both impairment and disability-oriented and provides more definitive information about the potential subject's appropriateness regarding movement abilities. It is used to determine subject appropriateness for any one of the three $\mathrm{CI}$ projects depending on their level of disability and balance deficits. While the PT screening evaluation focuses mainly on movement abilities, the subject is also screened for cognitive and/or communication deficits that would interfere with their participation as a research subject due to inability to understand and follow the instructions for the various motor assessment tests. Finally, a research assistant administers the MAL to the client. This portion of the screening evaluation is of importance as the subject must not exceed a maximum score of 2.5 ; thereby demonstrating that a significant disability is present. If the subject passes the physician's medical screening, meets the motor criteria for one of the projects, and does not score above a mid-range score on the MAL, they are accepted as a research subject for the appropriate project.

In summary, potential reasons for exclusion include motor ability that is too high or too low, cognitive deficits that prevent adequate participation, and an already high level of use of the more affected UE. While subjects failing to meet the three projects' inclusionary criteria are not accepted for the project, the criterion limits for subjects appropraite for CI movement therapy have yet to be established. Present and future studies will attempt to more clearly identify these criteria.

\subsection{Testing}

A battery of tests are conducted with all participants in the CI project. Results from some of these tests are used to test specific research hypotheses while others are used for diagnostic purposes and/or to generate new hypotheses. Tests routinely administered include the following.

\subsubsection{The wolf motor function test (WMFT)}

The WMFT, as presently used by our research team, consists of 18 motor tasks which examine contributions from the distal and proximal musculature of the UE tested. With few exceptions, the tasks are sequenced according to joint movements and muscle activity required; proximal to distal, gross to fine motor. Most tasks take place with the subject sitting in a chair placed at (depending on the task) specified distances from a table. The chair position may be adjusted based on each subject's height and physical characteristics (i.e. arm length). When chair position is altered, the change is recorded and repeated during post-test- 
ing. A diagrammed grid or template is taped to the desk to specify standardized measurements (i.e. placement of objects, excursion distances).

The WMFT is administered prior to intervention, immediately following intervention, and 2 years following intervention. Task items are described and demonstrated to subjects three times before they are asked to perform the task. Subjects are not allowed to practice the task as the instructions are given. Following the prompt of 'ready, set, go', they are asked to perform each task as quickly as possible and to attempt each task even if they do not believe they can complete the task. If the subject is unable to complete a tested task in the time allotted $(2 \mathrm{~min})$, the tester records a time of $120 \mathrm{~s}$ plus and moves on to the next task item in the test. For most tasks, performance time and a functional ability score are recorded. Only performance time is recorded for a grip release task. Weight lifted and pounds of force are recorded for a lift weight from table top to a 10-inch high to box and grip strength tasks, respectively. All attempts are videotaped for scoring by blind raters at a later time. Unlike early studies, the quality of movement rating scale has been dropped from the WMFT procedure. The investigative team made this decision after discovering a very high positive correlation $(>0.99)$ between the quality of movement and functional ability ratings. Therefore, it was believed that information gathered from the quality of movement scale would be redundant.

The investigative team was recently confronted with the problem of use of the WMFT for the testing of lower functioning subjects. The team anticipated that persons recruited for the VA project, due to their more significant disability status, might be unable to complete many of the WMFT items in the 2 min allotted. The resulting lack of data points would make analysis of this test data for these subjects difficult. Therefore, pilot testing procedures with these subjects also included the upper extremity portion of the Fugl-Meyer Test for Motor Function. A decision regarding using the WMFT for these subjects is still pending. A modified version of the test for lower functioning patients was developed previously and may be employed in the future.

\subsubsection{The motor activity $\log (M A L)$}

The MAL was developed for the purpose of exploring functional activities attempted outside of the clinical setting. As such, it explores the critical issue of transfer from the experimental to everyday home situation. We believe that this characteristic makes the MAL a truly unique functional assessment tool. The test's original version included questions about 14 specific tasks commonly carried out in the life situation. The test is a self report instrument administered in an interview format by one of the project staff members. In the interview, subjects are asked to rate their performance on each activity, emphasis being placed on performance at home. The test is administered on approximately 10 occasions and regarding different times frames including: (1) activity during the year prior to testing (but more than 1-year post-stroke); (2) activity during the week prior to testing; (3) activity during each day of the intervention period (half of the MAL is administered each day of the intervention period for a total of five complete MAL administrations during the intervention period); (4) once per week for the first month post-intervention; and (5) 2 years post-intervention.

\subsubsection{Range of motion measures}

Passive and active range of motion is measured immediately before and after the intervention period. Passive range of motion is measured first and is often filmed to be used as part of the Fugl-Meyer Test of Motor Function Assessment. While no specific hypotheses have been posed regarding the influence of $\mathrm{CI}$ on range of motion, we are monitoring this specific impairment for such an influence. Additionally, information gathered from the range of motion measures is critically important in identifying more impaired joints and movements. Subsequently, project staff are equipped to develop the most appropriate behavioral contract and shaping program (both described in more detail later in this paper).

\subsubsection{The actual amount of use test (AAUT)}

Our investigative team believes that differences often exists between how well a patient can use a more affected upper extremity when requested to 
do so when performing a laboratory motor test, and when actually using it in the life situation. Therefore, we have designed a test to obtain an unobtrusive measure of actual amount of use of the more affected upper extremity. The AAUT is first conducted when the subject first arrives at the research laboratory on their first testing day just prior to treatment. The AAUT is performed in front of a videocamera mounted on the wall and angled such that it views a majority of the room. The subject is unaware that they are being filmed, though they have already given informed consent permitting such activities. The tester unobtrusively prompts the subject to carry out a set of predetermined activities that are natural seeming and appropriate for an introduction session (e.g. flipping/leafing through a folder of project-related material, folding a piece of paper containing project information, putting paper in their wallet or purse, etc.). No indication is given on how tasks should be executed (i.e. which UE should be used). The subject is told that the purpose for the activities are to familiarize them with the laboratory setting. The AAUT is carried out prior to and immediately after the intervention period. Videotaped sessions are scored by raters who are blind to the subject's group or treatment status. Ratings are given regarding the amount of use of and quality of movement of the more affected UE using a six-point Likert scale.

\subsubsection{Cognitive tests}

Subjects participating in our project complete a battery of cognitive tests including the Mini Mental State Exam, the Sentence Repitition Section of The Multilingual Aphasia Examination, The Cancel $\mathrm{H}$ Test, The Token Test, The Zung Depression Scale, and a Visual Neglect Test. A minimal score of 19 on the Mini Mental State Exam is required for participation in the project. While no formal hypotheses have been posed regarding the influence of $\mathrm{CI}$ procedures on cognitive parameters, data gathered from these tests are being examined to see if exposure to the project appears to influence such characteristics. This is viewed as a type of control procedure. If contact with a project and the intervention procedure succeeded in improving cognitive function (toward which it is not directed) the question would arise whether any improvement in motor function was due to a generalized placebo-type effect.

\subsection{Assuring patient compliance}

The therapeutic procedures conducted under supervision in the clinic are only effective if the subject continues to use the more affected arm in their home environment. At the same time, however, we believe that subject safety should receive considerable attention as the restraint apparatus will reduce their ability to use their stronger UE during more complex, and potentially hazardous activities. Therefore, our project protocol includes several steps to improve subjects' understanding of and compliance with their therapeutic program. First, to assure that the subject will wear the hand splint/arm sling or half glove, they must be able to put on and take off the apparatus independently. A substantial portion of the first day is devoted to learning this task. To assist the subjects, all restraint equipment have been recently designed to allow easy placement and removal (i.e. velcro straps).

Once independence with restraint apparatus management is demonstrated, a behavioral contract is executed. Each subject first provides a detailed description of their typical daily routine starting with when they get up in the morning and ending with when they go to bed at night. Subjects are asked to provide as much detail as possible including approximate times these activities take place. For example, 08:00-08:30 - eat breakfast (cereal, toast, coffee, juice); 08:30-08:40 - brush teeth; 08:40-09:10 - take a shower; etc. From this daily schedule, a project staff member, usually a physical therapist, provides suggestions regarding which activities should be executed with: (1) the more affected UE only (while wearing the prescribed restraint device); (2) with both UEs; and (3) with the less affected UE only. Activities placed in the less affected UE only category are those posing safety risks to the subject (e.g. walking on stairs, handling hot liquids). The agreed-upon activities are written on the standardized behavioral contract form and signed 
by the subject, project staff member, and a witness. We believe that by formally executing this contract, we highlight the importance of the agreement and, subsequently, enhance compliance for many subjects. Several items commonly appear on subjects' behavioral contracts. Using the more affected UE for eating is almost always included. Tasks may be broken up into subtasks and placed on different list within the behavioral contract. For example, subjects may be asked to 'cut up food' with the both UEs, yet, 'manipulate a fork to bring food to their mouth' with the more affected UE only. Subjects are formally questioned about their compliance with the behavioral contract by completing a home diary with a project staff member on a daily basis.

The home diary outlines the patient's activities from the time they left the research laboratory until the time they return for that days session. All activities are discussed, not just items listed on the subject's behavioral contract. Subjects are asked to provide details regarding how the activities were executed (i.e. successfully or unsuccessfully, with or without assistance, with or without the restraint apparatus). While collecting this information, project staff members may remind the subject about the terms of the behavioral contract and/or provide suggestions to improve compliance. Information provided by the subject may lead the project staff member to modify the behavioral contract (e.g. move an activity into another category, add a new activity to the contract, etc.).

Immediately following the home diary exercise, the MAL is administered. While care is taken not to prompt specific answers on the MAL, remembering activities encountered, as is done during the home diary exercise, often assists subjects to provide more accurate MAL information.

\subsection{Day-to-day clinical procedures}

\subsubsection{Daily schedule}

Generally, subjects arrive at the research laboratory at 09:00 and leave at 15:00. Project staff record a detailed schedule of all clinical activities carried out each day of the intervention (Fig. 3). This schedule includes time devoted to each activity listed. The schedule specifically notes the times when the restraint device is put on and taken off. Also, the time and length of rest periods is also included. Specific shaping task practice is listed on the daily schedule. Further details regarding the tasks shaped are documented on a shaping data sheet (described below). Eating lunch with the more affected UE is particularly emphasized for all subjects. A daily record is kept not only of the length of time devoted to eating lunch, but also what foods were eaten and how this was accomplished. Information recorded on the daily schedule is particularly helpful during research team meetings and for providing possible explanations for post-intervention results.

\subsubsection{Shaping techniques}

Our investigative team believes that the behavioral training technique of shaping is an effective form of CI therapy; especially when used in conjunction with restraint of the less affected arm. Shaping has been used extensively as a behavioral training technique. Using shaping, a desired motor or behavioral objective is approached in small steps, by successive approximations. Shaping is very similar to training techniques commonly used by physical and occupational therapists giving patients task practice. The main difference is that, with shaping, patients or subjects are given explicit feedback concerning even small improvements in performance. Additionally, particular care is taken to select appropriate tasks that can be broken down into subtasks that can be objectively measured and where even small improvements are noticeable. A battery of approximately 60 tasks have been developed with a preliminary shaping plan for each. However, each subject's shaping program is individualized; selecting from the basic battery of 60 tasks and creating new ones when it seems that it would be advantageous to that subject's restaint program. The selection of shaping tasks for each individual depends on: (1) specific joint movements that exhibit the most pronounced deficit; (2) joint movements that project staff believe had the greatest potential for improving; and (3) the subjects preference among tasks that have a similar potential for producing 


\section{DAILY SCHEDULE}

Name: $\quad \underline{\text { Mr. Smith }}$

Time

$9: 15$

$9: 25$

$9: 45$

$10: 05$

$10: 30$

$10: 40$

$11: 10$

$11: 38$

$12: 15$

$12: 52$

1:00

2:00

2:04

$3: 14$

$3: 35$
Date: $\quad \underline{12 / 17 / 96}$

Activity

Home diary - sling off to drink coffee

MAL administered

Sling on

Velcro checkers - Shaping task 1

Stretching with PT

Light switch - Shaping task 2

Cotton balls - Shaping task 3

Large peg board - Shaping task 4

Lunch: Ate over $1 / 2$ of lunch with more affected UE, including tough corned beef, mashed potatoes, cabbage, and roll; moved cup with more affected UE.

Lunch continued with sling off

Rest break - sling off

Sling on

ARC and rings - Shaping task 5

Open and shut locker door - Shaping task 6

Fork and meat - Shaping task 7

Leave for home

Fig. 3. Example of daily schedule in the clinical setting. specific improvements. An example of a commonly used shaping task is provided below [28].

\subsubsection{Rotation of rolodex file}

A Rolodex file (12.7-cm diameter) is rotated by a seated subject by turning one of two knobs (5.7 $\mathrm{cm}$ in diameter) protruding from the center of either side of the file. The movement required is grasp (of the knob) and ulnar deviation and some flexion of the wrist. The arm of the subject is unsupported and kept in flexion at the shoulder and elbow. All joints of the UE are involved in the activity. A thin piece of red tape is secured across the radius of the knob and continued (with 
an interruption) onto the frame of the file. A trial begins with the segments of tape on the knob and frame lined up; completion of one rotation is indicated when the two segments are again lined up. The project staff member supervising the task practice informs the subject of performance progress by counting completed rotations out loud. The parameters shaped are number of rotations per $30 \mathrm{~s}$ and quality of movement.

When practicing the shaping tasks, modeling and prompting from the therapist are provided liberally. If needed, the subject may even be physically assisted to carry out part of the task they could not do by themselves. This 'assisted movement', however, is reduced and eventually faded out as soon as feasible. Verbal feedback regarding performance is provided enthusiastically after detecting even small improvements. Performance regressions are never punished and are usually ignored. If performance continues to exhibit no improvements after approximately three trials, the subject is gently encouraged to improve further (e.g. 'Let's see if you can do better next try'). If the subject continues to have difficulty, a simpler subtask, or entirely different task, is substituted.

Specific information about each shaping task is recorded on a shaping data sheet. Information recorded includes a description of the task, specific trial attempted, objective score from that trial, and additional comments. Scores obtained are graphed to provide visual feedback to the subject and project staff members regarding progress in performance throughout the intervention period.

\subsubsection{Team communication}

The investigative team meets for $1-1.5 \mathrm{~h}$ on a weekly basis. Team members in attendance usually include the projects principal investigator, medical director, physical therapists, and research assistants. At this time, results from specific screening evaluations are discussed and decisions made regarding acceptance of potential subjects into the project. In addition, the team discusses the progress of subjects already enrolled. At times, more global methodological issues are raised and discussed. In fact, many of the projects guiding hypotheses have been identified and discussed during these meetings. These meetings provide important brainstorming and problem solving opportunities. The investigative team views these weekly communication opportunities as critical to the project's success.

\subsubsection{Home practice}

During the last few days of the the $\mathrm{CI}$ intervention, an individualized home program is established for each subject. The investigative team believes that continued practice is important to maintain and possibly continue to improve results obtained during clinical intervention. In fact, a few of our study subjects have demonstrated significant improvement from immediately post-intervention to the 2-year follow-up. Like the CIT clinical procedures, home programs are task oriented. Commonplace tasks using easily obtained equipment are identified for each subject. These tasks will emphasize use of the most impaired movements and joints. Equipment used includes items typically found in the home setting (i.e. styrofoam cups for stacking) or easily purchased. Childrens toy stores are often used for equipment purchase. For example, ring toss and work bench toys have been incorporated into subject home programs in the past. Occasionally, impairment oriented exercises are included in subjects' home programs. As with clinical procedures, stretching and positioning exercises are more likely to be included. Strengthening and endurance exercises are rarely, if ever, included in home programs. Subjects are given written instructions regarding the home program and practice it under the supervision of project staff until independence is achieved.

\subsection{Other considerations}

\subsubsection{Treatment of impairments}

The CIT approach could be described as primarily task oriented in orientation. As such treatment is focused on treating patient problems at a disability level (i.e. emphasizing functional activities). At times, however, specific attention is placed on the treatment of patient problems at an impairment level (i.e. stretching specific muscles). Most typically, treatment of this type is focused on stretching soft tissue rendered non-compliant 
secondary to hypertonicity or increasing range of motion at restricted joints. Specifically, the projects physical therapists may perform passive range of motion and stretching exercises when the subject arrives in the morning to facilitate improved task practice. CIT procedures rarely focus on specific strengthening or endurance activities. We believe that these specific impairments are positively influenced indirectly through task practice. In fact, previous CIT studies indicate that dramatic improvements in strength were demonstrated in post intervention laboratory tests of motor function [28].

\subsubsection{Involvement from significant others}

Cooperation from the subjects' significant other or caregiver is also important for safety and compliance. A Patient/Caregiver Agreement is executed in conjunction with the behavioral contract. This agreement identifies one individual as responsible for being present when the subject is away from the research laboratory and practicing agreed upon tasks. Specific steps are taken to assure that his individual is acquainted with the subject's behavioral contract and the projects procedures. This agreement also states that the subject will remove the sling/splint or mitt if the caregiver is not present and the task poses safety concerns. The agreement is signed by the subject, caregiver and project staff member.

Significant others are also provided instruction regarding the subject's post-intervention home program. When possible, this individual will be present while this home program is practiced under project staff supervision. The investigative team believes that understanding of the home program by the significant other facilitates compliance with the home program. In other words, the significant other will hopefully provide additional encouragement and feedback to the subject, if necessary.

Finally, family members/caregivers are included in the project by completing the MAL with regards to their significant other's motor performance. The MAL is administered to the significant others on two occasions and concerning four time periods: (1) on the pre-treatment testing day - regarding the past week, period since the project evaluation, and period between the project evaluation and 1 year earlier; and (2) on the post-treatment testing day - regarding the subject's motor activity on that day (i.e. what has been the effect of the intervention?).

\subsubsection{Future direction of research}

To date, CIT has been explored for use with chronic stroke patients, meeting specific inclusionary criteria, and regarding UE motor function. In theory, however, the learned non-use phenomenon may extend to many different situations. For example, the mechanism could be applied to other portions of the body (i.e. lower extremity motor function). Patients experiencing disability related to other medical diagnoses may also experience learned non-use (i.e. spinal cord injury, extremity fractures). As a method of overcoming learned non-use, CIT could be modified and applied to the treatment of a variety of medical problems. Additionally, optimal times for CIT application have yet to be identified. For example, could CIT prevent the development of learned non-use if applied during more acute stages of rehabilitation? Finally, different modes of CIT delivery warrant investigation. For example, could CIT be administered effectively in the home setting or in a group format? Clearly, CIT has potential for improving motor function in patients recovering form a number of medical conditions.

\section{Acknowledgements}

This research was supported by Grant B93629AP from the Rehabilitation Research and Development Service, US Department of Veterans Affairs to Rama D. Pidikiti, Grant 94-172 from the Retirement Research Foundation and a grant from the Center for Aging, University of Alabama at Birmingham to Edward Taub and by a portion of the Emory University Research and Training Grant 008300041, from NIDRR, Department of Education, awarded to Steve Wolf. The authors would also like to acknowledge the following research team members: Francilla Allen, Anjan Chattergee, Leslie H. Cude, David J. Edwards, Stacy Goode, Sharon Shaw, Gitendra Uswatte, and Shari Yakley. 


\section{References}

[1] Taub E, Miller NE, Novack TA, Cook EW, Fleming $\mathrm{WD}$, Nepomuceno CS et al. Technique to improve chronic motor deficit after stroke. Arch Phys Med Rehabil 1993;74:347-354.

[2] Taub E, Crago JE. Overcoming learned non-use. A new behavioral approach to physical medicine. In: Kikuchi T, Sakuma H, Saito K, Tsuboi K, editors. Tokyo: SpringerVerlag, 1995:2-9.

[3] Taub E, Pidikiti RD, DeLuca SC, Crago JE. Effects of motor restriction of an unimpaired upper extremity and training on improving functional tasks and altering brain behaviors. In: Toole J, editor. Imaging and neurologic rehabilitation. New York, NY: Demos Publications, 1996:133-154.

[4] Taub E, Wolf SL. Constraint induction techniques to facilitate upper extremity use in stroke patients. Top Rehabil, 1997.

[5] Wolf SL, Lecraw DE, Barton LA, Jann BB. Forced use in hemiplegic upper extremities to reverse the effect of learned non-use among chronic stroke and head injured patients. Exp Neurol 1989;104:125-132.

[6] Andrews K, Stewart J. Stroke recovery: he can but does he? Rheumatol Rehabil 1979;18:43-48.

[7] Ernst E. A review of stroke rehabilitation and physiotherapy. Stroke 1990;21:1081-1085.

[8] de Pedro-Cuestra J, Widen-Holmquist L, Back-y-Rita P. Evaluation of stroke rehabilitation by randomized controlled studies: a review. Acta Neurol Scand 1992;86:433-439.

[9] Bach-y-Rita P. Recovery from brain damage. J Neurol Rehabil 1993;6:191-199.

[10] Bach-y-Rita P, Wicab Bach-y-Rita E. Biological and psychosocial factors in recovery from brain damage in humans. Can J Psychol 1990;44:148-165.

[11] Knapp HD, Taub E, Berman AJ. Effect of deafferentation on a conditioned avoidance response. Science $1958 ; 128: 842-843$

[12] Knapp HD, Taub E, Berman AJ. Movement in monkeys with deafferented forelimbs. Exp Neurol 1963;7:305-315.

[13] Taub E. Motor behavior following deafferentation in the developing and motorically mature monkey. In: Herman R, Grillner S, Ralston PS, Stein PSG, Stuart D, editors. Neural control of locomotion. New York, NY: Plenium Press, 1976:605-705.

[14] Taub E. Movement in nonhuman primates deprived of somatosensory feedback. In: Exercise and sports science reviews, vol. 4. Santa Barbara, CA: Journal Publishing Affiliates, 1977:335-374.
[15] Taub E, Bacon R, Berman AJ. The acquisition of a trace-conditioned avoidance response after deafferentation of the responding limb. J Comp Physiol Psychol 1965;58:275-279.

[16] Taub E, Berman AJ. Avoidance conditioning in the absence of relevant proprioceptive and exteroceptive feedback. J Comp Physiol Psychol 1963;56:1012-1016.

[17] Taub E, Ellman SJ, Berman AJ. Deafferentation in monkeys. Effect on conditioned grasp response. Science 1966;151:593-594.

[18] Taub E, Goldberg IA, Taub PB. Deafferentation in monkeys: pointing at a target without visual feedback. Exp Neurol 1975;46:178-186.

[19] Skinner BF. The behavior of organisms. New York, NY: Appleton-Century-Crofts, 1938.

[20] Skinner BF. The technology of teaching. New York, NY: Appleton-Century-Crofts, 1968.

[21] Risely TR, Baer DM. Operant behavior modification; the deliberate development of behavior. In: Caldwell M, Riccuiti HN, editors. Review of child development research, vol. III. Development and social action. Chicago, IL: University of Chicago Press, 1973:283-329.

[22] Morgan GW. The shaping game: a technique. Behav Ther 1974;5:481-488.

[23] Panyan MV. How to use shaping. Lawrence, KS: $\mathrm{H}$ and H Enterprises, 1980.

[24] Taub E. Somatosensory deafferentation research with monkeys: implications for rehabilitation medicine. In: Ince LP, editor. Behavioral psychology in rehabilitation medicine: clinical applications. New York, NY: Williams and Wilkins, 1980:371-401.

[25] Taub E, Perella PN, Miller EA, Barro G. Diminution of early environmental control through perinatal and prenatal somatosensory deafferentation. Biol Psychiatry 1975;10:609-626.

[26] Taub E, Miller NE, Novack TA, Cook EW, Fleming WD, Nepo Muceno CS, Connell JS, Crago JE. Technique to improve chronic motor deficit after stroke. Arch Phys Med Rehab 1993;74:354.

[27] Kopp B, Kunkel A, Flor H, Platz T, Ulrike R, Mauritz $\mathrm{KH}$, Gresser K, McCulloch KL, Taub E. The arm motor ability test (AMAT): Reliability, validity, and sensitivity to change of an instrument for assessing disabilities in the activities of daily living. Arch Phys Med Rehabil 1997; in press.

[28] Taub E, Crago JE, Burgio LD, Groomes TE, Cook EW, DeLuca SC et al. An operant approach to rehabilitation medicine: overcoming learned non-use by shaping. J Exp Anal Behav 1994;61:281-293. 